\title{
Studying the relative bottom contribution via heavy-quark decay electron measurements in STAR
}

\author{
André Mischke ${ }^{\mathrm{a}}$ \\ for the STAR Collaboration \\ Institute for Subatomic Physics, Faculty of Science, Utrecht University, Princetonplein 5, 3584 CC Utrecht, The Netherlands
}

Received: 27 August 2008 / Revised: 4 December 2008 / Published online: 7 February 2009

(C) Springer-Verlag / Società Italiana di Fisica 2009

\begin{abstract}
We report measurements of the azimuthal angular correlation distribution of heavy-quark decay electrons and open charmed mesons in $p p$ collisions at $\sqrt{s}=200 \mathrm{GeV}$ in the STAR experiment at RHIC. This measurement in combination with current theoretical model calculations allows to extract the relative bottom contribution to the heavy-quark decay electrons, which is important for the interpretation of the observed strong suppression of the high- $p_{\mathrm{T}}$ electron yield in central $\mathrm{Au}+\mathrm{Au}$ collisions.
\end{abstract}

\section{Introduction}

Measurements at the Relativistic Heavy Ion Collider (RHIC) at Brookhaven National Laboratory have revealed strong modification of the jet structure in high-energy nuclear collisions due to the interaction of hard scattered partons with the hot and dense matter created in these reactions (for a recent review, see [1]). The study of heavy-quark (charm and bottom) production offers unique opportunities for the investigation of the properties of the QCD matter under extreme conditions. Heavy quarks are believed to be produced predominantly in hard scattering processes in the early stage of the collision $[2,3]$. Due to their higher mass, the penetrating power is much higher for heavy quarks than for light quarks, providing a sensitive probe of the matter. The energy loss of heavy quarks in the medium is expected to be smaller compared to light-quarks and gluons due to the mass dependent suppression of the gluon radiation at small angles, the socalled dead-cone effect $[4,5]$.

Charm and bottom quarks are currently identified by assuming that isolated electrons in the event stem from semileptonic decays of heavy-quark mesons. At high transverse momentum $\left(p_{\mathrm{T}}\right)$, this mechanism of electron production is dominant enough to reliably subtract other sources of elec-

a e-mail: a.mischke@uu.nl trons like conversions from photons and $\pi^{0}$ Dalitz decays. STAR measurements in central $\mathrm{Au}+\mathrm{Au}$ collisions have shown that the high- $p_{\mathrm{T}}$ yield of electrons from semileptonic charm and bottom decays is suppressed relative to properly scaled proton-proton collisions, usually quantified in the nuclear modification factor $\left(R_{A A}\right)$ [6]. This factor exhibits an unexpectedly similar amount of suppression as observed for light-quark hadrons, suggesting substantial energy loss of heavy quarks in the produced medium. Energy-loss models incorporating contributions from charm and bottom do not describe the observed suppression sufficiently well [7, 8]. Although it has been realized that energy loss of heavy quarks by elastic parton scattering causing collisional energy loss is probably of comparable importance to energy loss by gluon radiation $[9,10]$, the quantitative description of the suppression is still not satisfying. Furthermore, it has been shown that fragmentation of heavy quarks into heavy mesons which are collisionally dissociated, a process that can happen multiple times in the medium, may be significant in heavy-ion collisions [11]. Only theoretical models which include energy loss from charm alone describe the observed suppression reasonably well [8].

Recent calculations of heavy-flavor production in $p p$ collisions at Fixed-Order plus Next-to-Leading Logarithm (FONLL) level have shown that the bottom contribution to the heavy-quark decay electrons (so-called non-photonic electrons) is significant at intermediate $p_{\mathrm{T}}(4-5 \mathrm{GeV} / c)$ although the uncertainties are relatively large [12]. Since both $D$ and $B$ decays contribute to the non-photonic electrons, these relative contributions have to be determined experimentally.

In this contribution, measurements of azimuthal angular correlations of heavy-quark decay electrons and open charmed mesons in $p p$ collisions at $\sqrt{s}=200 \mathrm{GeV}$ are presented, which yield important information about the underlying production mechanism. Requiring $e-D^{0}$ coincidence in the same event significantly improves the signal- 
to-background ratio over either technique individually. The shape of the azimuthal correlation distribution provides a more differential comparison between the charm and bottom contributions due to their different decay kinematics.

\section{Data analysis}

The $p p$ data used for this analysis were recorded at $\sqrt{s}=$ $200 \mathrm{GeV}$ by the STAR Time Projection Chamber (TPC) [13] and the barrel electromagnetic calorimeter (BEMC) [14] in Run VI. The TPC identifies charged particles through their ionization energy loss and provides precise tracking in a magnetic field of $0.5 \mathrm{~T}$ over a large kinematical range $(|\eta| \leq$ 1.8 with full azimuthal symmetry) with very good momentum resolution. The BEMC is a lead-scintillator sampling calorimeter with a radiation length of $21 \mathrm{X}_{0}$ and an energy resolution of $\delta E / E \approx 16 \% / \sqrt{E}$. The calorimeter, situated behind the TPC, is divided into 4800 tower cells and covers an acceptance of $|\eta|<1$ and full azimuth. Inside the BEMC, at a depth of $\sim 5.6 X_{0}$, is a shower maximum detector (SMD) which consists of two layers of gas wire pad chambers. The SMD provides measurements of the profile of electromagnetic showers and the position of the shower maximum with high resolution $((\Delta \eta, \Delta \phi)=(0.007,0.007))$. The BEMC served as a leading electron or photon trigger to study high$p_{\mathrm{T}}$ particle production. The integrated luminosity sampled by the BEMC high-tower trigger is $11 \mathrm{pb}^{-1}$ by applying an energy threshold of $E_{T}=5.4 \mathrm{GeV}$ for the highest energy in a BEMC cell.

Electrons are identified by combining the information from the TPC (particle momentum and ionization energy loss $(d E / d x))$ and the BEMC (cell energy). In contrast to hadrons, electrons deposit most of their energy in the BEMC cells. A cut on the shower profile size combined with a requirement on the ratio momentum-to-cell energy, $0<p / E<2$, rejects a large amount of hadrons. The final electron sample is obtained by applying a momentum dependent cut on the ionization energy loss within the range $3.5<d E / d x<5.0 \mathrm{keV} / \mathrm{cm}$ to optimize the electron efficiency and hadron rejection (hadron suppression factor is $10^{5}$ at $p_{\mathrm{T}}=2 \mathrm{GeV} / c$ and $10^{3}$ at $p_{\mathrm{T}}=7 \mathrm{GeV} / c$ ). The measured electrons have two main contributions: One is from heavy-flavor decays, the other from photon conversions in the detector material between the interaction point and the TPC $\left(X / X_{0} \sim 4.5 \%\right)$ and $\pi^{0}$ and $\eta$ Dalitz decays. The latter is typically called "photonic electrons". These photonic electrons are identified statistically and rejected based on the invariant mass method [6].

Open charmed mesons are reconstructed via their hadronic decay $D^{0} \rightarrow K^{-} \pi^{+}(B R=3.89 \%$ [15]) by calculating the invariant mass of all oppositely charged TPC track of particles in the same event. The negative track of particles have to fulfill a $d E / d x$ cut of $\pm 3 \sigma$ around the expected kaon band to enhance the kaon candidate probability. In this analysis, only events with a non-photonic electron trigger are used for the $D^{0}$ reconstruction, which suppresses significantly the combinatorial background of random pairs. Furthermore, the kaon candidates have to have the same charge sign as the non-photonic electrons (called like-sign electron-kaon pairs). The resulting invariant mass distribution of $K$ and $\pi$ pairs shows a pronounced $D^{0}$ peak around the expected value. The invariant mass distribution without a non-photonic electron trigger does not have a $D^{0}$ signal for the applied track quality cuts. Consequently, the requirement of a non-photonic electron trigger suppresses the combinatorial background significantly, leading to a signalto-background ratio of $\sim 14 \%$ and a signal significance of $\sim 3.7$ [16].

\section{Results and discussion}

The invariant mass distribution of $K$ and $\pi$ pairs is obtained for different $\Delta \phi$ bins with respect to the trigger electron, and the yield of the associated $D^{0}$ mesons is extracted as the area underneath a Gaussian fit to the signal. Figure 3.1 (left panel) shows the azimuthal angular correlation distribution of non-photonic electrons and $D^{0}$ mesons, which exhibits a near- and away-side correlation peak with similar yields. In the following, we imply electron $-D^{0}$ and positron $-\overline{D^{0}}$ pairs when using $e-D^{0}$.

The data are compared to results from PYTHIA and MC@NLO simulations [17]. The observed away-side correlation peak can be attributed to prompt charm pair production $(\sim 75 \%)$ and $B$ decays $(\sim 25 \%)$, whereas the near-side peak, by contrast, represents essentially contributions from $B$ decays.

The relative bottom contribution to the non-photonic electrons $(B /(B+D))$ has been extracted using two approaches. Firstly, by requiring like-sign $e-K$ pairs which selects bottom on the near-side and charm on the away-side (cf. Fig. 3.2). The relative bottom contribution is found to be $0.57 \pm 0.23$ and $0.52 \pm 0.22$ for the PYTHIA and MC@NLO simulations, respectively. Secondly, the relative bottom contribution is determined from the measured $D^{0}$ yields on the away-side which selects charm for like-sign $e-K$ pairs and bottom for unlike-sign $e-K$ pairs (cf. Fig. 3.2). The $B /(B+D)$ ratio is $0.43 \pm 0.23$. The results, summarized in Fig. 3.1 (right panel), agree with measurements of the azimuthal angular correlations of non-photonic electrons and charged hadrons [18]. Moreover, the data show agreement with the FONLL calculation within the model uncertainties [12].

In summary, heavy quarks are identified and separated on a statistical basis through their characteristic decay topology 


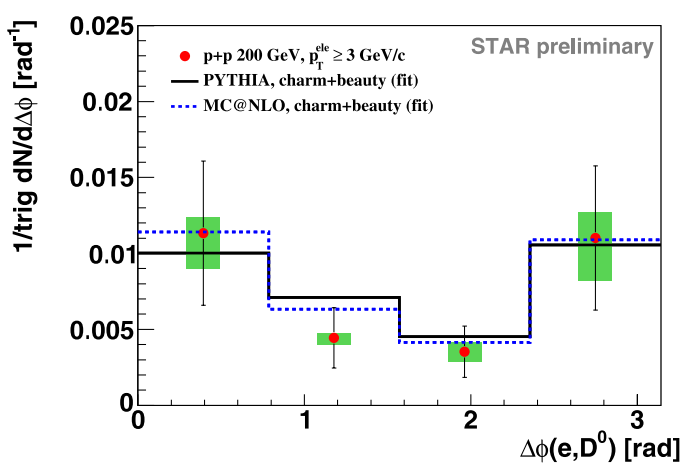

Fig. 3.1 (Color online) Left panel: Azimuthal angular correlation distribution of non-photonic electrons and $D^{0}$ mesons (for like-sign $e-K$ pairs) in $p p$ reactions at $\sqrt{s}=200 \mathrm{GeV}$. The green boxes indicate the systematic uncertainties. The black/solid (blue/dashed) histogram is the result from PYTHIA (MC@NLO) simulations fitted to the data.

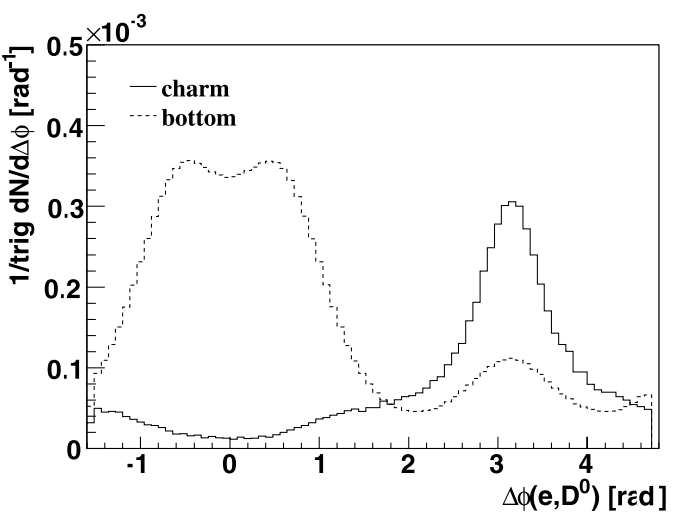

Fig. 3.2 Azimuthal angular correlation function of electrons and $D^{0}$ mesons from charm (solid curve) and bottom decays (dashed curve) generated in PYTHIA simulations of $200 \mathrm{GeV} p p$ reactions requir-

using azimuthal angular correlation of non-photonic electrons and identified $D^{0}$ mesons. The relative bottom contribution to the non-photonic electrons in $200 \mathrm{GeV} p p$ collisions has been extracted using different methods. The data, which are in agreement with results from $e$-hadron correlation measurements from STAR and recent FONLL calculations, indicate that bottom decay yields are of comparable magnitude at and above $\sim 4 \mathrm{GeV} / c$.

Acknowledgements The author would like to thank the organizers for the stimulating atmosphere during the conference. This work was supported by a Veni grant from the Netherlands Organization for Scientific Research (project number 680-47-109).

\section{References}

1. R. Stock, arXiv:0807.1610 (2008)

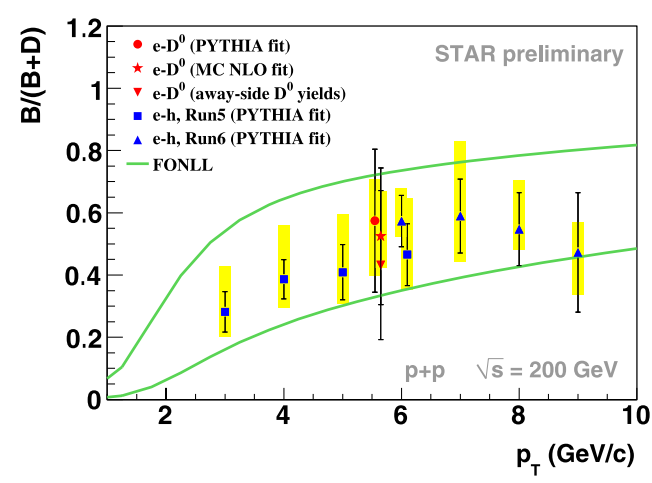

Right panel: Relative bottom contribution to the non-photonic electron yield derived from different fits to the $e-D^{0}$ azimuthal correlation distribution (red symbols). The data are compared to $e$-hadron correlations (blue symbols) and to the uncertainty band from a FONLL calculation (green curves). The yellow boxes depict the systematic uncertainties

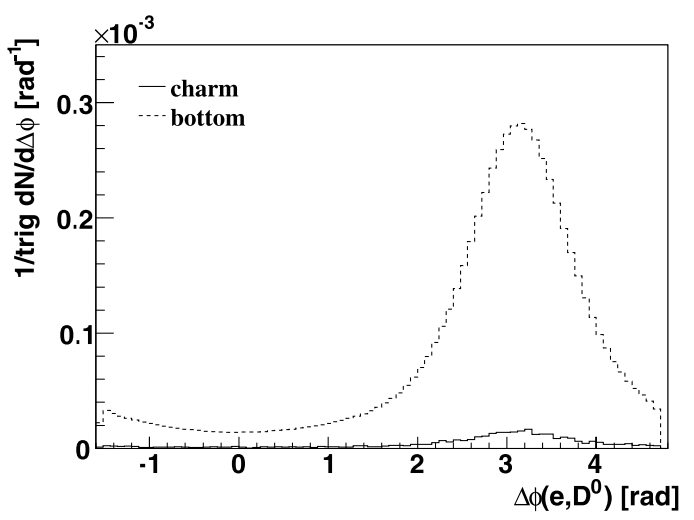

ing like-sign (left panel) and unlike-sign $e-K$ pairs (right panel). The transverse momentum of the trigger electrons is $3<p_{\mathrm{T}}<7 \mathrm{GeV} / c$. For details see [17]

2. Z. Lin, M. Gyulassy, Phys. Rev. C 51, 2177 (1995)

3. X. Dong et al., These Proceedings

4. Y.L. Dokshitzer, D.E. Kharzeev, Phys. Lett. B 519, 199 (2001)

5. M. Djordjevic, M. Gyulassy, S. Wicks, Phys. Rev. Lett. 94, 112301 (2005)

6. B.I. Abelev et al., Phys. Rev. Lett. 98, 192301 (2007)

7. M. Djordjevic, M. Gyulassy, R. Vogt, S. Wicks, Phys. Lett. B 632, 81 (2006)

8. N. Armesto, M. Cacciari, A. Dainese, C.A. Salgado, U.A. Wiedemann, Phys. Lett. B 637, 362 (2006)

9. S. Wicks, W. Horowitz, M. Djordjevic, M. Gyulassy, Nucl. Phys. A 784, 426 (2007)

10. H. van Hees, V. Greco, R. Rapp, Phys. Rev. C 73, 034913 (2006)

11. A. Adil, I. Vitev, Phys. Lett. B 649, 139 (2007)

12. M. Cacciari, P. Nason, R. Vogt, Phys. Rev. Lett. 95, 122001 (2005)

13. M. Anderson et al., Nucl. Instrum. Methods A 499, 659 (2003)

14. M. Beddo et al., Nucl. Instrum. Methods A 499, 725 (2003)

15. C. Amsler et al. (Particle Data Group), Phys. Lett. B 667, 1 (2008)

16. A. Mischke et al., J. Phys. G: Nucl. Part. Phys. 35, 044022 (2008)

17. A. Mischke, Phys. Lett. B 671, 361 (2009)

18. G. Wang et al., J. Phys. G: Nucl. Part. Phys. 35, 104107 (2008) 